\title{
On the determination of the atmospheric outer scale length of turbulence using GPS phase difference observations: the Seewinkel network
}

\author{
Gaël Kermarrec ${ }^{1^{*}}$ (D) and Steffen Schön ${ }^{2}$
}

\begin{abstract}
Microwave electromagnetic signals from the Global Navigation Satellite System (GNSS) are affected by their travel through the atmosphere: the troposphere, a non-dispersive medium, has an especial impact on the measurements. The long-term variations of the tropospheric refractive index delay the signals, whereas its random variations correlate with the phase measurements. The correlation structure of residuals from GNSS relative position estimation provides a unique opportunity to study specific properties of the turbulent atmosphere. Prior to such a study, the residuals have to be filtered from unwanted additional effects, such as multipath. In this contribution, we propose to investigate the property of the atmospheric noise by using a new methodology combining the empirical mode decomposition with the Hilbert-Huang transform. The chirurgical "designalling of the noise" aims to filter both the white noise and low-frequency noise to extract only the noise coming from tropospheric turbulence. Further analysis of the power spectrum of phase difference can be performed, including the study of the cut-off frequencies and the two slopes of the power spectrum of phase differences. The obtained values can be compared with theoretical expectations. In this contribution, we use Global Positioning System (GPS) phase observations from the Seewinkel network, specially designed to study the impact of atmospheric turbulence on GPS phase observations. We show that (i) a two-slope power spectrum can be found in the residuals and (ii) that the outer scale length can be taken to a constant value, close to the physically expected one and in relation with the size of the eddies at tropospheric height.
\end{abstract}

Keywords: Outer scale length of turbulence, GPS phase observations, Double difference residuals, Matérn model, Kolmogorov spectrum

\section{Introduction}

The Global Navigation Satellite System (GNSS) refers to constellations of satellites that transmit microwave signals in the L-band. The four GNSS are the European Galileo, the US Global Positioning System (GPS), the Chinese Beidou and the Russian GLONASS system to which can be added local systems such as the QZSS in Japan or NavIc in India (Hoffmann-Wellenhof et al.

\footnotetext{
*Correspondence: kermarrec@gih.uni-hannover.de

${ }^{1}$ Geodetic Institute, Leibniz Universität Hannover, Nienburger Str. 1,

30167 Hannover, Germany

Full list of author information is available at the end of the article
}

2008). The signals transmitted are affected by the Earth's atmosphere; Particularly the signals are delayed and refracted in the troposphere- a non-dispersive medium for microwaves that extends from the ground to approximately $10 \mathrm{~km}$ (Stull 1994). Contrary to the ionosphere, its impact on a GNSS signal cannot be mitigated or eliminated by linear combinations (Leick et al. 2015; Teunissen and Montenbruck 2017). The delay in the zenith direction called the Zenith Total Delay has two major components: the Zenith Hydrostatic Delay and the Zenith Wet Delay (ZWD). Whereas the hydrostatic delay can be accurately modelled (see, e.g., Saastamoinen 1973), the 
ZWD is a major error source in GNSS positioning, particularly for the Up component (Beutler et al. 1987; Dodson et al. 1996). This wet delay is often estimated with the least squares (LS) method as an additional parameter together with the position (Klos et al. 2018), and is an important data source in meteorology (Bevis et al. 1994; Douša and Vaclavovic 2014). It can be used to determine the distribution of atmospheric water vapour for shortterm weather and rainstorm prediction (Champollion et al. 2004; Choy et al. 2013; Li and Deng 2013; Zhao et al. 2018) and for urban applications, such as flooding events (Champollion et al. 2009; Guerova et al. 2016). Long periodic variations of tropospheric wet delays are caused mainly by the steady-state component of the refractive index field.

On the other hand, stochastic variations of the refractive index are induced by tropospheric turbulence that occurs along the signal's path from the transmitter to the receiver (Naudet 1996; Schön and Brunner 2008; Vennebusch and Schön 2011). An accurate knowledge of this second-order effect due to the variations of the refractivity index is of main importance for the higher temporal resolution of water vapour variations. These variations affect microwave phase and phase difference measurements by correlating them (see, e.g., Hinder 1972; Stotskii and Stotskaya 1992; Buscher et al. 1995 for interferometric observations; Schön and Brunner 2008; Kermarrec and Schön 2014 for GPS; Halsig et al. 2016 for very long baseline interferometry). The observations are no longer stochastically independent: one value recorded at time $t$ will have some similarity with a value taken at $t+\tau, \tau$ being a time increment and at a different location separated by a spatial distance $d r$. With a wavelength of approximately $20 \mathrm{~cm}$, GNSS phase measurements are affected by atmospheric turbulence (Tatarskii 1971; Wheelon 2001). Regarding physical considerations, the anisotropic turbulence of the free atmosphere from $1000 \mathrm{~m}$ above the Earth's surface is expected to correlate with the phase observations, contrary to the isotropic and well-mixed turbulence of the boundary layer (Myrup 1969; Wheelon 2001).

Residuals from GNSS relative positioning with phase or phase difference observations, e.g., by differencing measurements between receivers to a common satellite, contain precious information about the turbulent atmosphere to assess the second-order effect of the refractive index: a deep analysis of the residuals allows an estimation of some model parameters, such as power law exponents or cut-off frequencies, to be made. The power spectrum density (psd) of the phase difference observations can be physically modelled from theoretical investigations by combining concepts from turbulence theory with electromagnetic wave propagation in a random medium (Ishimaru 2017; Wheelon 2001 and the references therein). Thus, we expect to find a superposition of a fractional Gaussian noise (fGn) with a power law of $-2 / 3$ and a Matérn process with a smoothness of $-4 / 3$ in observation residuals.

Prior to such an estimation from GNSS observations, the geometric part of the observations has to be separated from the stochastic part by adequate processing. A relative positioning scenario, based on so-called double differencing (DD), is often chosen to extract as many systematic effects as possible (Schön and Brunner 2008). Residuals from other positioning scenario, such as Precise Point Positioning or single differencing, need a connection to an ultra-stable external oscillator in order to be interpretable (Schön et al. 2016). Unfortunately, multipath (due to multiple reflections of the signals, see Leick et al. 2015) is responsible for repetitive low-frequency patterns, which bias the power law and cut-off estimation of the tropospheric noise. A mitigation or elimination of such additional effects has to be performed in a preprocessing step to avoid this unwanted effect. We will use the empirical mode decomposition (EMD) in this contribution (Hirrle 2017); as opposed to wavelet decomposition (see, e.g., Satirapod and Rizos 2005), the EMD breaks down the residuals into various components empirically. It allows the fGn (Flandrin et al. 2004) to be extracted with a high reliability because it is independent of predefined basis functions. Thereafter, the correlation parameter of the remaining noise can be estimated and compared with theoretical expectations (see Wheelon 2001, chapter 6).

We will use observations from a network specially designed for estimating turbulent parameters from GPS phase difference residuals to validate the expected theoretical noise model and validate our methodology (Schön and Brunner 2008). The cut-off between the slopes can be accurately estimated using the Whittle maximum likelihood (Sykulski et al. 2019); this allows the computation of the outer scale length of turbulence, which we will interpret based on the separation distance between GPS rays. A further application of this result is the proposal of an adequate stochastic model for GNSS observations to be integrated in the LS adjustment to avoid a problematic overestimation of the precision of GNSS positioning results. We note that the methodology developed in this contribution can be used for other kind of geodetic time series processed using double differenced GNSS phase observations, such as ZWD (Klos et al. 2018).

The remainder of the paper is structured as follows: in a first part, we will briefly present the concept of DD positioning, the second part is devoted to the description of the expected noise, and a third part presents the results 
for the estimation of the outer scale length of turbulence from the Seewinkel network.

Note Throughout the paper, we will use the terminology "GNSS" when the derivations apply to all systems and GPS for results that were specifically tested with the observations under consideration.

\section{Global navigation satellite system carrier phase observation}

\section{Concept of relative positioning}

GNSS satellites transmit microwave signals in the L-band for positioning and timing. The carrier phase signal between a satellite $k$ and a user $A$ can be modelled by to $10 \mathrm{~km}$ (see Fig. 1). Thus, atmospheric delays will be largely reduced, as well as the impact of phase wind und and satellite antenna offsets. Receiver clock errors, satellite clock errors, and hardware delays are eliminated.

We mention further that the major part of the first-order ionospheric effect on GPS observation can be removed using the so-called ionosphere-free linear combination of L1 and L2 phase observations (see, e.g., Odijk 2002). It is defined as $\Phi_{\text {ionofree }}=\left(f_{1}^{2} \Phi_{\mathrm{L} 1}-f_{1}^{2} \Phi_{\mathrm{L} 2}\right) /\left(f_{1}^{2}-f_{2}^{2}\right)$, where $\Phi_{\mathrm{L} i}, i=1,2$ indicates measurements in the frequency $f_{1}=1575.42 \mathrm{MHz} f_{2}=1227.60 \mathrm{MHz}$. This additional frequency will be used complementary to validate the results.

$$
\Phi_{A}^{k}=\rho_{A}^{k}+c\left(\delta t_{A}-\delta t^{k}\right)+T_{A}^{k}-I_{A}^{k}+M_{A}^{k}+\lambda N_{A}^{k}+\cdots+\varepsilon_{A}^{k},
$$

where $\rho_{A}^{k}$ denotes the Euclidian distance between the unknown user position and the computed satellite position at the time of signal transmission, $c\left(\delta t_{A}-\delta t^{k}\right)$ the synchronization error between the satellite and receiver clock, and $T_{A}^{k}, I_{A}^{k}$ the tropospheric delay and ionospheric signal advance, respectively. Further corrections must be applied for, e.g., antenna phase centre corrections and phase wind up. The wavelength of the signal is denoted by $\lambda$ and the ambiguity is $N_{A}^{k}$, the multipath effect is described by $M_{A}^{k}$ and the observation noise of typical 1-2 mm by $\varepsilon_{A}^{k}$. Interested readers should refer to the basics of GNSS positioning in the textbooks (HofmannWellenhof et al. 2008; Leick et al. 2015; Teunissen and Montenbruck 2017).

Equation (1) underlines the duality between the signal and the nuisance parameter: if the position is known, GNSS signals can be used to study the other terms. Finally, if all terms are corrected or eliminated by combinations of observations, the noise can be studied either with pre-fitted residuals (sometimes called observed minus computed values) or with post-fit residuals after LS adjustment. We will use observed minus computed values in this contribution in order to avoid a potential coupling of the observation residuals due to the adjustment.

Double differences between four carrier phase observations $\Phi_{A}^{k}, \Phi_{B}^{k}, \Phi_{A}^{j}, \Phi_{B}^{j}$ are formed to reduce even eliminate error contributions, depending on their similarity-the DD considering two stations $A$ and $B$ and two satellites $j$ and $k$ reads:

$$
\mathrm{DD}_{A B}^{j k}=\left(\Phi_{B}^{k}-\Phi_{A}^{k}\right)-\left(\Phi_{B}^{j}-\Phi_{A}^{j}\right) .
$$

The elevation of the two satellites at the two stations as well as the atmospheric conditions in the free atmosphere will be nearly identical for medium baseline lengths of up
Finally, the DD time series still contains:

- A multipath arising from multiple reflections of the signal on the ground. A far- or near-field multipath will cause mostly low components that can be identified in the residuals (Leick et al. 2015).

- Flicker noise (FN) or white noise (WN) comes from electronic devices, cables, receivers or emitters themselves, and

- the atmospheric noise (AN) in which we are interested.

In this contribution, we propose to perform a filtering of these additional effects with the EMD and the

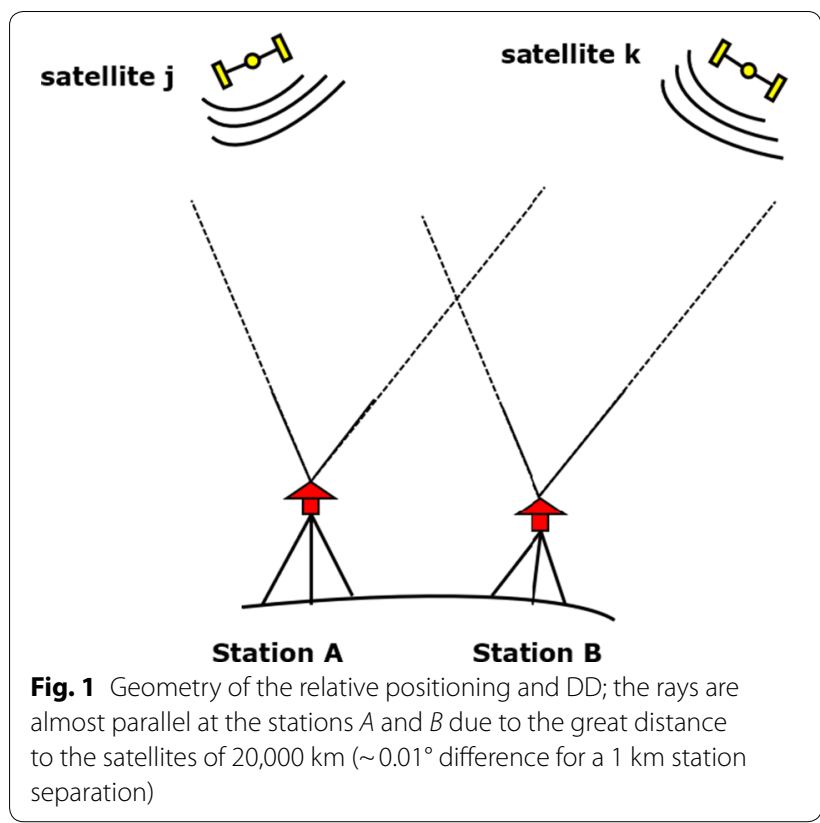


corresponding intrinsic mode functions (IMF). We will follow results from Flandrin et al. (2004) on the fGn, which will be used to model-in its damped versionthe atmospheric or tropospheric noise that affects GNSS phase differences.

\section{Fractional Brownian motion and fractional Gaussian noise}

Fractional Brownian motion ( $\mathrm{fBm})$ is a self-similar Gaussian process with stationary increments and is a mathematical generalization of the classical random walk and Brownian motion. The term "fractional" is related to the fractional integration and differentiation (Lilly et al. 2017). The fBm has a long-range, strong spatial and temporal correlation. Its increment is called an fGn and was introduced by Mandelbrot and Van Ness (1968) to face the lack of derivative of an $\mathrm{fBm}$. The $\mathrm{fGn}$ is a generalization of ordinary WN: its statistical properties are determined by its second-order structure. More precisely, the power law decay of the fGn spectrum is controlled by a single scalar parameter, called the Hurst exponent $H$, with $0<H<1$. For the special case $H=1 / 2$, the fGn reduces to a WN. For $1 / 2<H<1$, the long-range correlations are said to be persistent, which is the case of interest in this contribution. The power-law exponent $\beta$ of the spectrum of the process $W(f) \infty 1 / f^{\beta}$, with $f$ being the frequency, is related to $H$ by $H=(\beta+1) / 2$ for an fGn.

\section{Empirical mode decomposition \\ Principle}

Huang et al. (1998) developed the EMD to decompose a given time series into a set of components of different time scales called the IMF. Combined with the HuangHilbert Transform (hht), this decomposition provides a time-frequency-energy representation of a signal, which is intuitively based on the wave form of the data. It has been used successfully in various domains, from seismic detection to the correction of orbital drift artefacts in satellite data stream, ocean waves or signal analysis, to cite but a few (see Huang and Shen 2005 and the references within). A major field of applications of EMD is to denoise a signal, and various strategies have been proposed to reach that goal. We cite exemplarily regarding geodesy the extraction of WN from GPS co-ordinates time series (Montillet et al. 2013), thresholding-based methods to extract seismic waves (Wang et al. 2012) and mitigation of multipath effects (Wu 2009; Hirrle 2017).

The EMD is an iterative method of low computational complexity, in which spurious harmonics are less likely to appear: no linear property is imposed on a possibly nonlinear system. Its empirical approach is independent of mother wavelets-as in wavelet analysis-or of some trigonometric functions-as in Fourier transform. The EMD when used on non-stationary signals, allows a direct extraction of the local energy associated with the IMF. Its main drawback is linked to the non-uniqueness of the decomposition; the latter is related to a particular interpolation scheme for envelope extraction, the stopping criterion (usually $1 \mathrm{e}^{-6}$ ) used in the sifting process and the number of IMF chosen.

The EMD algorithm is summarized in Flandrin et al. (2004) as the following loop:

- identification of the extrema of the signal.

- interpolation between minima (maxima) to get an envelope of the signal.

- computation of the average of the envelope.

- extraction of the detail as the difference between the envelope and the original signal.

- iteration on the residuals.

An IMF should also have the following properties: (i) zero mean, (ii) all the maxima and all the minima of one IMF are correspondingly positive and negative, and (iii) narrowband. Each IMF has fewer extrema than all the lower order IMFs, leading to decreasing oscillations as the IMF order increases.

The original temporal signal $S(t)$ is finally a nonweighted combination of all the IMF obtained.

$$
S(t)=\sum_{i=1}^{K} d_{i}(t)+m_{K}(t),
$$

where $d_{i}(t)$ is the $i$ th IMF and $m_{K}(t)$ the residuals after $K$ decomposition.

A variance $\sigma_{\mathrm{IMF}, i}^{2}$, corresponding to its mean energy, is associated to each IMF. Under "energy", we understand the empirical variance estimate of a given IMF.

\section{Intrinsic mode functions of a fractional Gaussian noise}

The IMF of an fGn and their corresponding energies are studied in detail in Flandrin et al. (2004) and Wu and Huang (2004) for the specific WN case $(H=1 / 2)$. The authors estimated the psd of each mode using dedicated simulations and showed that the EMD is acting as a dyadic filter bank of constant-Q band pass filters on the fGn.

Apart from the first noise-only IMF, which carries most of the WN component and, thus, the main energy, the variance of the other IMF $d_{i}(t), i=2 \ldots K$ decreases linearly in a semi-log diagram regarding the IMF index.

Decomposition of the fGn: slope of the IMF variance To illustrate this property, we simulated three fGn, each of length 60,000, corresponding to a Hurst parameter of 0.5 (WN), 1 (FN, red line) and 0.83 (atmospheric fGn, AN, see "The atmospheric noise from phase difference" sec- 
tion or Wheelon 2001). Please note that we will use these three abbreviations for the names of the different noises hereafter and for the sake of readability. We used the Matlab function ffgn to generate the time series (Stoev 2020). The latter are depicted in Fig. 2 (left), together with the IMF $\log _{2}$ variance (Fig. 2 right) estimated with the Matlab function hht and emd using seven decomposition steps. The theoretical expectation that the IMF variance should decrease exponentially as a function of the IMF index is confirmed. The corresponding negative slope $\kappa_{H}$, starting from the second IMF, gives an estimation $\hat{H}$ of the Hurst parameter:

$$
\hat{H}=1+\frac{\kappa_{H}}{2} .
$$

In the following, we will use the following notation: $\mathrm{IMF}_{i}$ is the $i$ th IMF, $i$ the index of the $i$ th IMF, i.e. $i=[1, \ldots, K]$, so that the $\log _{2}$ variance of the $i$ th IMF can be simply expressed as:

$$
\log _{2}\left(\sigma_{\mathrm{IMF}, i}^{2}\right)=\kappa_{H} i+C, i=2 \ldots K,
$$

$C$ being a constant.

Figure 2 (right) highlights further that:

- The slope of a noise combination consisting of AN and WN (magenta line) is between the one of a single WN (yellow line) and AN (red line) from $\mathrm{IMF}_{2}$.

- The slope for an FN is nearly 0 from $\mathrm{IMF}_{2}$ and is -1 for a WN. Thus, when the total noise is a combination of power law noises, the slope of the $\log _{2}$ vari- ance gives an indication of the dominant source, which may be FN, WN or AN.

- An AN "diluted" in a combination of WN and FN of equal variances (green line) leads to a change in the slope regarding a simple AN (red line) for a finite sample size. This change will depend on the respective variances of the different noises. The slope of the green line in our example is affected by the WN component up to $\mathrm{IMF}_{5}$.

- The $\log _{2}$ variance of $\mathrm{IMF}_{1}$ always behaves differently than the one of higher index and should not be integrated in the slope estimation. This is coherent with the empirical results of Flandrin et al. (2004, 2005).

\section{Proposal to extract atmospheric noise} First step: elimination of multipath/flicker noise signal

Any discrepancies from the linear relationship between the mean energy of the IMF and their index can be used to denoise a signal (see, e.g., or Wu and Huang 2004; Kopsinis and McLauglin 2009), as highlighted in Fig. 2 (right). In this contribution, however, we wish to analyse the noise and not the signal. We will use the terminology "designalling the noise" intentionally where the signal comes here from a multipath signature, additional noise, such as FN, or noise from functional misspecifications. Such a model was chosen to compute the position.

The first step of the procedure to extract the AN consists of estimating the slope $\hat{\kappa}_{H}$ from the mean energies of $\mathrm{IMF}_{i}, i \in[2,3,4]$, which is performed by regression. Based on the linear slope parameter $\hat{\kappa}_{H}$ and $C$ obtained (see Eq. (5)), we compute the standard error for
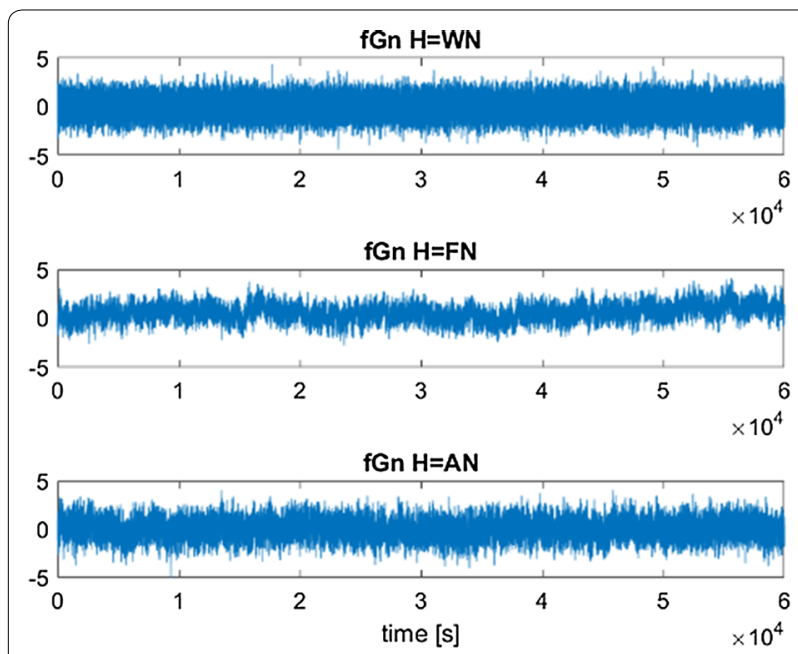

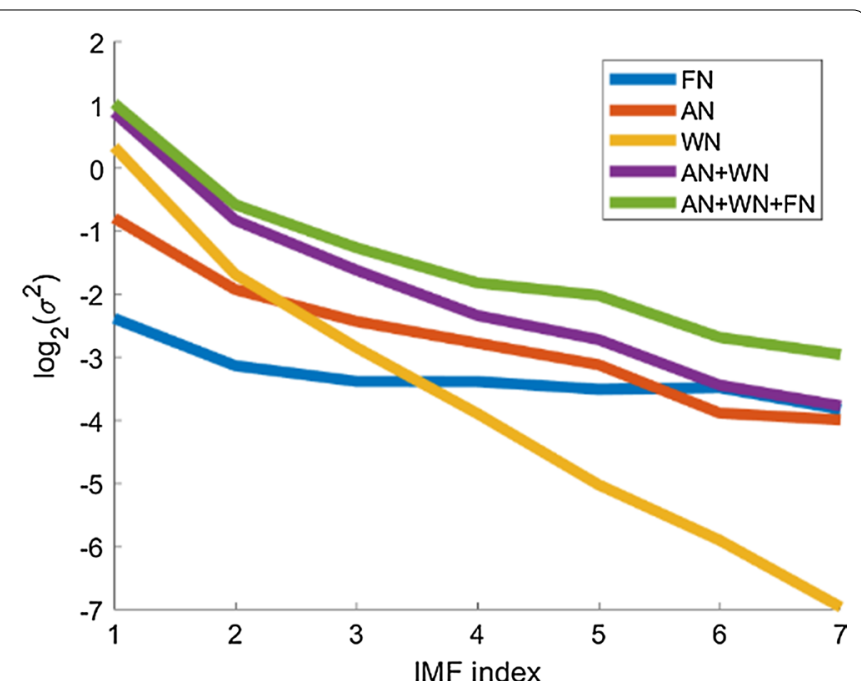

Fig. 2 Left: time series corresponding to the $\mathrm{fG} n$ with a different Hurst parameter (WN, FN and AN). Right: the corresponding $\log _{2}\left(\sigma_{\mathrm{IMF}, i}^{2}\right), i=1 \ldots 7$ for different noise combinations 
prediction $\Delta$, for which $\hat{\kappa}_{H} \pm \Delta$ corresponds to a roughly $68 \%$ prediction interval.

The strategy to filter the signal from the noise by excluding some IMF was firstly develop for WN. In this contribution, we extend it to an fGn, following the proposed methodology in Wu and Huang (2004) for WN. Consequently:

- We iteratively control whether $\log _{2}\left(\hat{\sigma}_{\mathrm{IMF}, i}^{2}\right)=\log _{2}\left(\sigma_{\mathrm{IMF}, i}^{2}\right) \pm 3 \Delta, i=5 \ldots K \quad$ to decide if the $i$ th IMF should be further considered or not. If not, the corresponding IMFs are no longer dominated by an fGn but rather by multipath, functional misspecifications or eventually noise with a higher power law than the AN (see Fig. 2, green line), i.e. what we called the "signal".

- The filtered $S_{\text {filt }}(t)$ is finally computed as:

$$
S_{\text {filt }}(t)=\sum_{i=1}^{4} d_{i}(t)+\left\{\sum_{i=5}^{K_{\text {filt }}} d_{i}(t)\right\},
$$

where $\left\{\sum_{i=5}^{K_{\text {filt }}} d_{i}(t)\right\}$ depends on the remaining number of IMF $K_{\text {filt }}$.

As suggested in Fig. 2 (right), the slope estimated may be affected when the noise is an unknown combination of different power law noises, as exemplarily, $\mathrm{AN}+\mathrm{WN}+\mathrm{FN}$. Following results from device design and analysis (Bahreyni 2008), we consider that the FN is to be found at low frequencies, i.e. in the higher IMF, and, thus, eliminated by the previous filtering. Such results are coherent with the previous comment made in Fig. 2. It remains, thus, to filter the WN from the remaining fGn $S_{\text {filt }}(t)$ in order to extract the AN only, which we perform using the EMD.

\section{White noise filtering}

From the last step, the high-pass filtering led to $S_{\text {filt }}(t)=\sum_{i=1}^{K_{\text {filt }}} d_{i}(t)$. We assume that this remaining noise can be decomposed into two components: $S_{\text {filt }}(t)=S_{\mathrm{WN}}(t)+S_{\mathrm{AN}}(t)$, where $S_{\mathrm{WN}}(t)$ is the WN and $S_{\mathrm{AN}}(t)$ the AN. Unfortunately, the WN component affects the power spectral density (psd) of $S_{\text {filt }}(t)$ strongly at high frequencies by driving its slope toward 0: an interpretation of $S_{\text {filt }}(t)$ as an AN is made meaningless without a prior whitening. In a first step, we start the filtering using the empirical maximization algorithm developed in Kargoll et al. (2018) in order to determine the ratio $R_{W N}$ of the standard deviation of the WN to the one of $S_{\text {filt }}(t)$. We further assume that the main part of the $\mathrm{WN}$ is present in the first IMF of the EMD decomposition, which contains most of the variance of $S_{\text {filt }}(t)$ (Flandrin et al. 2004; Wu and Huang 2004; Taebi 2017). Consequently, the WN is extracted from the first IMF by means of $S_{\text {filt }}(t)=R_{\mathrm{WN}} d_{1}+\left(1-R_{\mathrm{WN}}\right) d_{1}+\sum_{i=2}^{K_{\text {filt }}} d_{i}(t)$. The AN part is, thus, finally written as:

$$
S_{\mathrm{AN}}(t)=\left(1-R_{\mathrm{WN}}\right) d_{1}+\sum_{i=2}^{K_{\mathrm{filt}}} d_{i}(t) .
$$

This component will be further analysed based on the physical consideration developed in "The atmospheric noise from phase difference" section.

\section{Summary of the methodology to extract the atmospheric fractional Gaussian noise}

The final procedure to extract the $\mathrm{AN}$ is summarized in a flowchart form in Fig. 3. It involves three steps:

1. Computation and preprocessing of DD residuals (mean and trend extraction).

2. Elimination of the non-noise component (mainly multipath and low frequency FN).

- emd and hht decomposition.

- plot the $\log _{2}$ of the mean energy against $i \in[2,3,4]$, and visual inspection to validate the noise structure and dominant source (linear dependency).

- computation of the slope for $i \in[2,3,4]$ : the decision whether to integrate further IMF with $i \in[5, \ldots, K]$ is based on the criterium that $\log _{2}\left(\hat{\sigma}_{\mathrm{IMF}, i}^{2}\right)=\log _{2}\left(\sigma_{\mathrm{IMF}, i}^{2}\right) \pm 3 \Delta, i=5 \ldots K$.

3. Filtering of the WN component by downweighting the first IMF.

\section{Further comments}

Following Flandrin et al. $(2004,2005)$ and without loss of generality, we will fix $K=7$. We make use of the Matlab built-in function emd. The interpolation method chosen is pchip, which is dedicated to quick varying signals.

\section{The atmospheric noise from phase difference}

Once the AN has been extracted from the DD residuals, its correlation structure and phase spectrum can be analysed based on a physically plausible consideration. Consequently, we follow Wheelon (2001, chapter 6, p. 304-305). In a first subsection, we will briefly present the assumptions made, followed by a description of the two slopes noise model and its dependencies. 


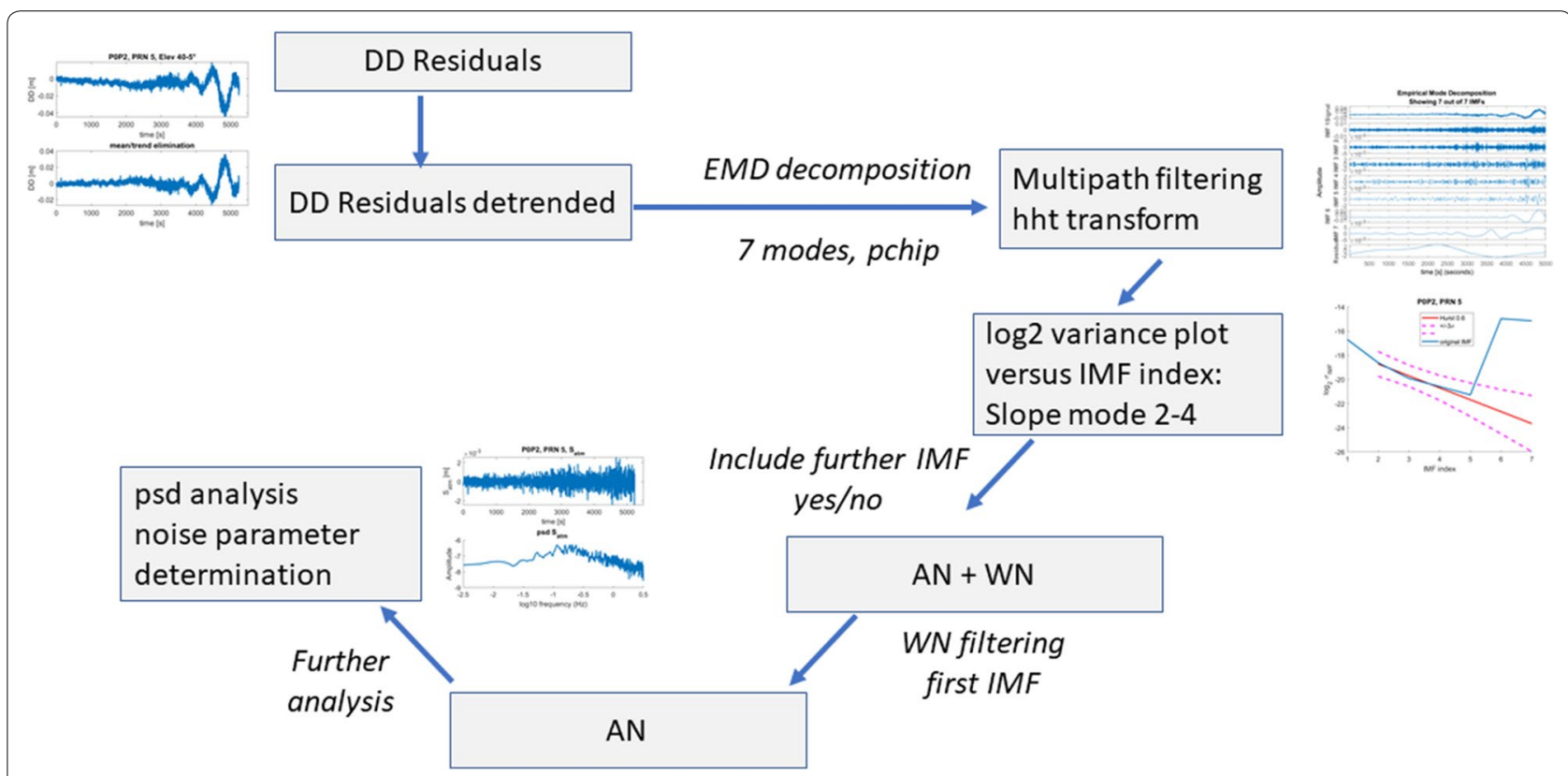

Fig. 3 The methodology to extract an AN in three steps

\section{The Matérn process or a damped fractional Brownian motion to model atmospheric noise Atmospheric temporal correlations}

Since turbulence theory is usually concerned with the statistical moments of the velocity or its fluctuations. Turbulent variations of the refractive index in the atmosphere can be modelled using the concept of eddies or swirl of motions (Stull 1994). These energy structures develop with wind shear, which is most often caused by microbursts from thunderstorms, temperature inversions, and surface obstructions. Eddies may have horizontal or vertical circulation. In the Earth's atmosphere, small eddies occur in the daytime when surface air is warmed by contact with the ground and then rises. On a larger scale, the high- and low-pressure centres that move over the Earth may be considered eddies, i.e. they are anomalous to the general circulation.

Consequently, the turbulent process is described using an energy cascade in which eddies subdivide into progressively smaller eddies until they are dissipated by viscosity or heat. The cascade starts at the outer scale length wavenumber and ends when eddies have the size of the inner scale length. Phase measurements are mainly influenced by large eddies, so that the main region of interest in the cascade is the energyinput region where the atmospheric wind field becomes unstable and creates large, two-dimensional energetic structures (Wheelon 2001).
Figure 4 proposes a schematic representation of the troposphere where eddies of different size and energy coexist. Eddies in the boundary layer are more likely to be small and isotropic with a high reorganization rate: the so-called three-dimensional turbulence. On the contrary, anisotropic eddies, elongated mainly in the horizontal direction, will be present in the loosely called free troposphere, from the tropospheric height at approximately $1000 \mathrm{~m}$ altitude (Stull 1994).

Using a geometric optics models and having in mind a positioning with DD processing, a ray coming from a GNSS satellite is expected to encounter the same elongated eddies as another ray coming from the reference satellite. This assumption holds true as long as the distance between the rays do not exceed the size of the largest eddies that affect the phase measurements. Figure 5 explains this concept schematically, highlighting that the rays that are spatially close (bold dotted and dotted black for the non-reference "green" satellite) will be temporally correlated. We call that effect "temporal correlations with spatial components".

\section{Modelization of the power spectrum for phase differences}

Many natural noises have approximate isotropic power spectra of the power-law form. From dimensional analysis, Kolmogorov (1941) has shown the power spectrum of passive scalars, such as temperature or refractive index can be written as: 

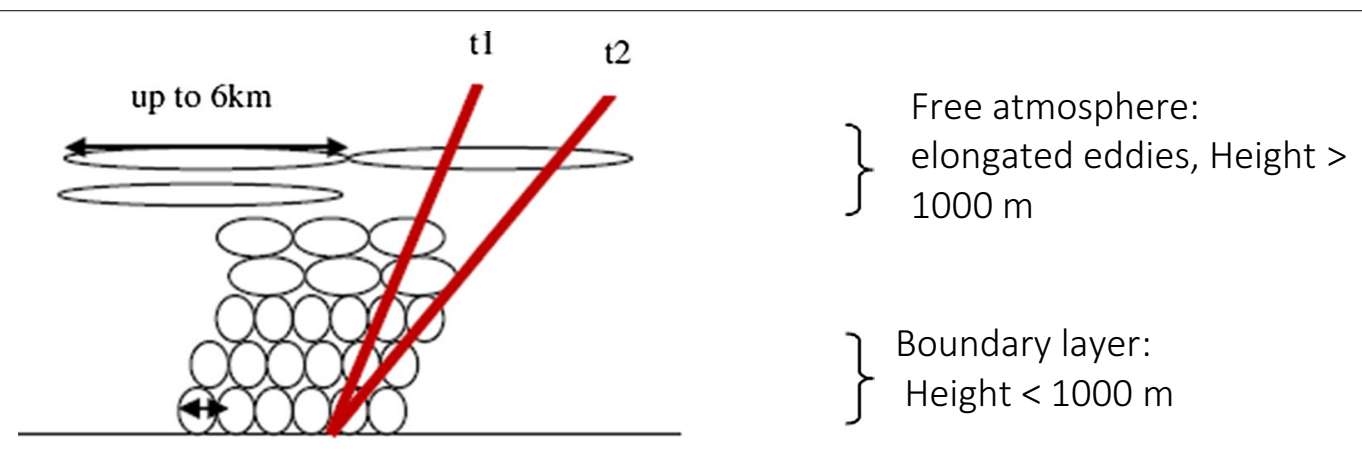

a few $\mathrm{mm}$

Fig. 4 GNSS signal path through the turbulent troposphere using the concept of isotropic eddies in the boundary layer and anisotropic two-dimensional structure in the free atmosphere. $t 1$ and $t 2$ are two epochs of measurements. The figure is adapted from Kermarrec and Schön (2014)

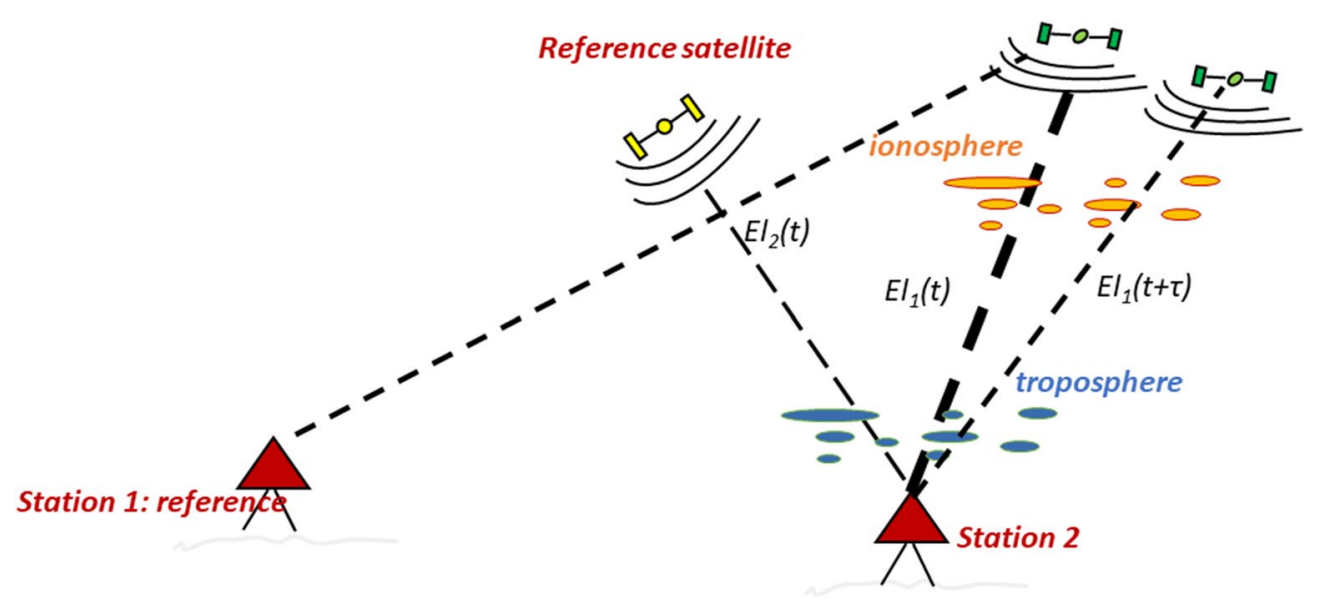

Fig. 5 Impact of the atmosphere on GNSS signals for a relative positioning scenario

$$
\Phi_{n}(\boldsymbol{\kappa})=\frac{0.033 C_{n}^{2}}{\left(\kappa_{x}^{2}+\kappa_{y}^{2}+\kappa_{z}^{2}\right)^{11 / 6}},
$$

where $C_{n}^{2}$ is the structure constant of the atmosphere and $\boldsymbol{\kappa}=\left[\begin{array}{lll}\kappa_{x} & \kappa_{y} & \kappa_{z}\end{array}\right]^{\mathrm{T}}$, the vector of three-dimensional wavenumbers. This model is valid in the inertial range for homogeneous and isotropic turbulence, where $2 \pi / L_{0} \leq \kappa \leq 2 \pi / l_{0}$ with $L_{0}, l_{0}$ being the outer and inner scale length of turbulence that bounds the inertial range, respectively.

In order to preserve the behaviour of the spectrum in the inertial range, the empirical Von Karman model is often preferred to the Kolmogorov description. It reads:

$$
\Phi_{n}(\boldsymbol{\kappa})=\frac{0.033 C_{n}^{2}}{\left(\kappa_{x}^{2}+\kappa_{y}^{2}+\kappa_{z}^{2}+\kappa_{0}^{2}\right)^{11 / 6}}
$$

with $\kappa_{0}$ being the wavenumber corresponding to a cutoff frequency that allows finite expressions for the first two moments of the spectrum. As mentioned previously, anisotropic eddies are more likely to correlate with the GNSS signals. Stretched rectangular co-ordinates can be used to account for the anisotropy and the spectrum is expressed as:

$$
\Phi_{n}(\kappa)=a b c \Phi_{n}\left(\sqrt{a^{2} \kappa_{x}^{2}+b^{2} \kappa_{y}^{2}+c^{2} \kappa_{z}^{2}}\right),
$$

where $a, b$ and $c$ are stretching factors describing the elongation of the eddies in the three dimensions. Usually, $a=b$ describes the horizontal elongation of the eddies, whereas $c$ is related to the vertical stretching.

The residuals of the DD processing of GNSS phase observations are time-dependent. Consequently, only the temporal correlations can be assessed: the spatial phase covariance between two GNSS rays from two satellites 
can be transformed into its temporal counterpart by means of the co-ordinate transformation $\mathbf{r}=\mathbf{r}^{\prime}-\mathbf{v}_{\text {eddies }} \tau$ using Taylor's frozen hypothesis (Taylor 1938), $\boldsymbol{r}^{\prime}$ and $\mathbf{r}$ being the position and the transformed position, respectively. With this approximation, eddies are considered to be frozen during the measurement, only moved by the mean wind $\mathbf{v}_{\text {eddies}}$, which is further assumed to blow parallel to the baseline. It can be replaced by a mean velocity of $v_{\text {eddies }}=8 \mathrm{~ms}^{-1}$, a good approximation at the top of the boundary layer (Stull 1994). This value corresponds to the geostrophic wind, which is a theoretical wind balanced by the Coriolis and pressure gradient forces. Under the approximation mentioned previously, we call $\rho_{\text {ray }}$, i.e. the Euclidian distance between 2 GNSS rays from the same satellite, computed at a given height.

Following Wheelon (2001, chapter 6) or Tatarskii (1971, chapter 4) and using the previous assumptions, the power spectrum of phase difference $\Delta \Phi=\Phi^{k}-\Phi^{j}$ between the phases of two satellites $k$ and $j$ can be derived from the single-path power spectra $W_{\Phi}(\omega)$ as

$$
W_{\Delta \Phi}(\omega)=4 \sin ^{2}\left(\frac{\omega \rho_{\text {ray }}}{2 v_{\text {eddies }}}\right) W_{\Phi}(\omega),
$$

where $\omega$ is the angular frequency. The factor $\sin ^{2}\left(\omega \rho_{\text {ray }} / 2 v_{\text {eddies }}\right)$ corresponds to the interference pattern of the receiver (Ishimaru 2017). By integrating along the lines-of-sight, $W_{\Phi}(\omega)$ is expressed as the product of a factor that accounts for the elevation of the satellite and a damped power law spectrum, i.e.

$$
W_{\Phi}(\omega)=2.192 H_{\text {trop }} \frac{k^{2} C_{n}^{2} c a^{-5 / 3} v_{\text {eddies }}^{5 / 3}}{\sin ^{2}(\mathrm{El})} \frac{1}{\left[\omega^{2}+\alpha^{2}\right]^{5 / 3}},
$$

where $\alpha=\kappa_{0} v_{\text {eddies }} / a=2 \pi v_{\text {eddies }} / a L_{0}$, El is the elevation of the satellite, $H_{\text {trop }}$ the tropospheric deep or height taken to $1000 \mathrm{~m}$ (Stull 2009), $a=b$ and $c$ the horizontal and vertical stretched parameters for the outer scale length, respectively, and $C_{n}^{2}$ is the structure constant, which varies with height but is here considered as a constant. $k$ is the electromagnetic wavenumber. $W_{\Phi}(\omega)$ corresponds to the power spectrum of a Matérn process, i.e. a damped fBm (Lilly et al. 2017). The cut-off frequency $\alpha=2 \pi v_{\text {eddies }} / a L_{0}$ is related to the wind velocity in the free atmosphere and the horizontal outer scale length of turbulence $a L_{0}$, which is known to be several kilometres along most of the path travelled by the GPS signals from the satellites to the receiver. We may conjecture from Fig. 3 that the cut-off frequency could correspond to the maximum size of the eddies that will correlate with the GPS signals between two epochs. The threshold angular frequency $\alpha=2 \pi v_{\text {eddies }} / a L_{0}$ is less than $0.01 \mathrm{~Hz}$ for $v_{\text {eddies }}=8 \mathrm{~ms}^{-1}$. When the time series of residuals are long enough to allow for a trustworthy estimation can only be estimated, i.e. at least 5000 epochs a $1 \mathrm{~s}$.

Following Wheelon (2001) and from Eq. (12), the spectrum of GNSS phase difference should behave as $\omega^{-2 / 3}$ below a corner frequency of $\omega_{c}=2 v_{\text {eddies }} / \rho_{\text {ray }}$, i.e. for $\sin ^{2}\left(\omega \rho_{\text {ray }} / 2 v_{\text {eddies }}\right) \sim\left(\omega / \omega_{c}\right)^{2}$.

Consequently, we expect to have two slopes in the psd of GPS phase difference residuals:

- At high frequencies, i.e. $\omega>\omega_{c}, W_{\Delta \Phi}(W) \approx 1 / \omega^{-8 / 3}$

- between, i.e. $\omega_{c}<\omega<2 \pi v_{\text {eddies }} / a L_{0}$, $W_{\Delta \Phi}(\omega) \approx 1 / \omega^{-2 / 3}$

- $\omega>2 \pi v_{\text {eddies }} / a L_{0}, W_{\Delta \Phi}(\omega) \approx 1 / \omega^{0}$.

Using this decomposition in three domains, we consider that the Matérn process corresponding to $W_{\Phi}$ will start at a high frequency as an fGn and will switch to a Matérn model at a lower frequency, i.e. a saturation.

Note on double differences we assume that the power spectrum of phase double differences in this contribution is the same as the power spectrum of phase differences. By making this approximation, we consider a "mean" station between $A$ and $B$ and take the difference between the phase from the same satellite $k$ (or $j$ ) at two stations, i.e. $\Phi(t)=\left(\Phi_{B}^{k}-\Phi_{A}^{k}\right)(t)$, as the phase measurement. The value $\rho_{\text {ray }}$ is not the distance between the two stations. We consider that the latter is not playing a role in the correlation structure, which we justify by the relatively long baseline length chosen in our case study regarding the value of the outer scale length of turbulence expected.

Note on ionospheric correlations ionospheric correlations due to plasma irregularities can be modelled with a similar power law spectrum as tropospheric turbulence (Rino 1979). Using GPS observations from Swarm satellites, Kermarrec et al. (2018) demonstrates that a similar model to that for tropospheric correlations can be used for ionospheric irregularities. However, the outer scale length is expected to be up to five times larger than in the free atmosphere and cannot be detected with the relatively short residuals samples of DD adjustment. That is the reason we allow ourselves to loosely use the terminology "atmospheric correlations" to describe correlations due to the turbulent variations of refractive index in the troposphere only. For the sake of completeness and to validate this approximation, we will use observations from the ionospheric-free linear combination in our real data analysis (Leick et al. 2015). 


\section{How to estimate the noise parameters The debiased Whittle maximum likelihood}

Dealing with real observations, the noise parameters of the damped $\mathrm{fBm}$ will have to be estimated from the preprocessed DD residuals. Three parameters of the correlation function have to be estimated from Eq. (12): the variance, the range and the smoothness. This estimation can be done with the maximum likelihood estimator: (i) as in the so-called Hector software (Bos et al. 2008) with an adapted version of the Matérn model-the Gauss Markov model-or (ii) with the powerful Whittle maximum likelihood estimator. This method leads to a fast and nearly unbiased estimation of the noise parameter for large samples (Sykulski et al. 2019). It further produces estimates that are asymptotically efficient and avoids biases coming from visual slope determination. Usual ML strategies involve matrix inversion: they are avoided when the autocovariance matrix is approximated in the frequency domain. Unfortunately, the so-called "Whittle likelihood" uses the periodogram of the observations, which may be a biased measure for finite samples due to blurring and aliasing effects (Percival and Walden 1993). Sykulski et al. (2019) proposed an alternative based on quasi-likelihood called the "de-biased Whittle likelihood", which is particularly interesting for discretetime processes of finite length, such as the residuals of the LS adjustment used in this contribution.

The Whittle likelihood in its discretized form is given by $l_{W}(H)=-\sum_{\omega \in \Omega}\left[\log (\tilde{f}(\omega, H))+\frac{I(\omega)}{\tilde{f}(\omega, H)}\right]$, with $\Omega$ the set of discrete Fourier frequencies, $f(\omega, H)$ the continuous-time process spectral density and $I(\omega)$ the perio$\operatorname{dogram} I(\omega) \infty \sum_{j=1}^{N}\left|z_{j} e^{-i j \omega}\right|^{2}$.

The Whittle estimator assumes a priori that the power spectrum of the underlying process of the dataset is known and should only be used if a time series is consistent with the process under consideration. In a first approximation, the visual inspection of the psd validates the presence of typical Matérn damping at low frequencies (see Lilly et al. 2017) or not.

In this contribution, we use the Whittle maximum likelihood estimator as implemented in Matlab by Lilly (2020).

\section{Parameters to be estimated}

From Eq. (12), we have to force for the estimation of a Matérn noise (see Lilly et al. 2017) corresponding to a smoothness of $4 / 3$ and a cut-off frequency $\omega_{c}=2 v_{\text {eddies }} / \rho_{\text {ray }}$. Furthermore, a second Matérn noise with a smoothness close to $1 / 3$ should be estimated from the residuals with a saturation at $\alpha=2 \pi v_{\text {eddies }} / a L_{0}$. This value is expected to be close to a constant, as it depends mostly on atmospheric parameters, or at least on the maximum size of the eddies that will correlate with the GNSS measurements. Unfortunately, the Matérn process is only defined for a smoothness higher than $1 / 2$, so that the estimator is constrained for a damped FN estimation.

\section{Real data analysis}

In this section, we validate the methodology using GPS observations from the Seewinkel network (Schön and Brunner 2008; Kermarrec and Schön 2014). We note that our methodology is not dependent on the GNSS system and can be extended for Galileo, Beidou, GLONASS or further satellites systems, provided that phase observations are used and processed using a relative positioning.

In this case study, we will show that an estimation of the cut-off frequency from the DD residuals is (i) feasible and (ii) provides physically plausible estimation. We further aim to analyse its dependencies.

The network will be presented briefly in a first subsection. A second part explains didactically the methodology and the computation of the outer scale length and cut-off frequency for one time series of residuals, which is extended to all visible satellites in a third subsection.

\section{Observations}

We use GPS phase observations from the Seewinkel network, which was specially designed to study the impact of temporal correlations due to turbulence (Schön and Brunner 2008). This network is located in a flat terrain in eastern Austria (Burgenland, 47.7 N, 17.0 E, 160 m ellipsoidal height) and consists of six aligned stations P0, P1, P2, P4, P8 and P16 with separations of approximately 1, 2, 4, 8 and $16 \mathrm{~km}$, respectively, measured on 15 April 2003 during $8 \mathrm{~h}$ (5:45-13:45 GPS time) using identical equipment, a $1 \mathrm{~Hz}$ data rate and a cut-off-angle of $3^{\circ}$ (see Fig. 6). We use the stations up to P8 so that the assumptions that DD are eliminating ionospheric refraction still holds. In this contribution, we use L1 observations (1575.42 MHz signal) without loss of generality and the ionospheric-free combination obtained from L1 and L2 (1227.60 MHz signal) additionally (Leick et al. 2015).

The GPS processing was done with the Bernese GPS Software Version 5.0 (Dach et al. 2009). In a first step, the coordinates of the stations P0 to P16 as well as relative troposphere parameters were precisely determined as a relative network w.r.t P0 using the ionosphere-free linearcombination L0. The ambiguities were fixed using the

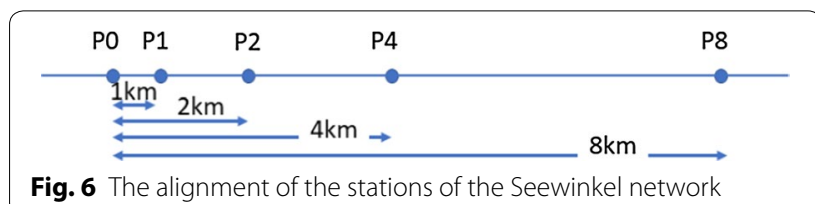


SIGMA strategy in a widelane-narrow-lane run. The coordinates of P0 were held fixed to a previously determined position w.r.t the GNSS station in Graz. In these computations, the whole available data material of ca. $8 \mathrm{~h}$ is used with a data rate of $30 \mathrm{~s}$. Precise final orbit and clocks products from the International GNSS Service (IGS, Johnston et al. 2017) were introduced as well as antenna correction.

In a second step, the coordinates of the stations were held fixed and the undifferenced OMC for different frequencies (L1, L2, L0) were computed sequentially for each station with the Bernese software for a $1 \mathrm{~s}$ data rate. Here, the 5-s CODE satellite clocks, final orbits and global ionospheric model were used (Dach et al. 2020). The receiver time scale was synchronized to GPS time by code observations and all typical corrections were applied, like antenna phase centre correction for user and satellite antennas from the IGS. Using a specially implemented writing option, the raw carrier phase observations as well as all corrections computed by Bernese were stored for $2 \mathrm{~h}$ data segments. This length allows for a reliable estimation of the correlation parameter.

In a last step-in own MATLAB code-the double differences were formed following Eq. (2), and the ambiguities solved by rounding the OMC to the nearest integer.

For the sake of shortness, we only present the results corresponding to P1 and P8, for which the North, East and $\mathrm{Up}(\mathrm{N}, \mathrm{E}, \mathrm{U})$ components are estimated. The skyplot corresponding to the observation time span is presented in Fig. 7. PRN6 was taken as the reference satellite for the DD computation (PRN or pseudorandom noise can be considered as the satellite number). An a priori standard deviation of $1 \mathrm{~mm}$ was assumed for the L1 carrier phase measurements.

Although the GPS observations may not correspond to the actual state of the art, the network is unique as the stations are ideally aligned. It allows to study the cut-off frequency predicted from atmospheric turbulence theory, which its designer Prof. F.K. Brunner was searching for. Further contribution will focus on more actual data set using, e.g., single differences.

\section{Didactical example}

\section{Preprocessing of the $D D$ residuals}

We use the DD between PRN5 and PRN6 from baseline P0P2 for our didactical example. This first batch of observations consists of 5050 epochs during which the satellites were visible. The elevation of PRN5 is between $40^{\circ}$ and $5^{\circ}$. The corresponding DD residuals are plotted in Fig. 8 (left). From epoch $3000 \mathrm{~s}$, we recognize the typical sinusoidal envelope corresponding to a strong far field multipath effect due to the decreasing elevation of the satellite: it has to be eliminated prior to the AN analysis.

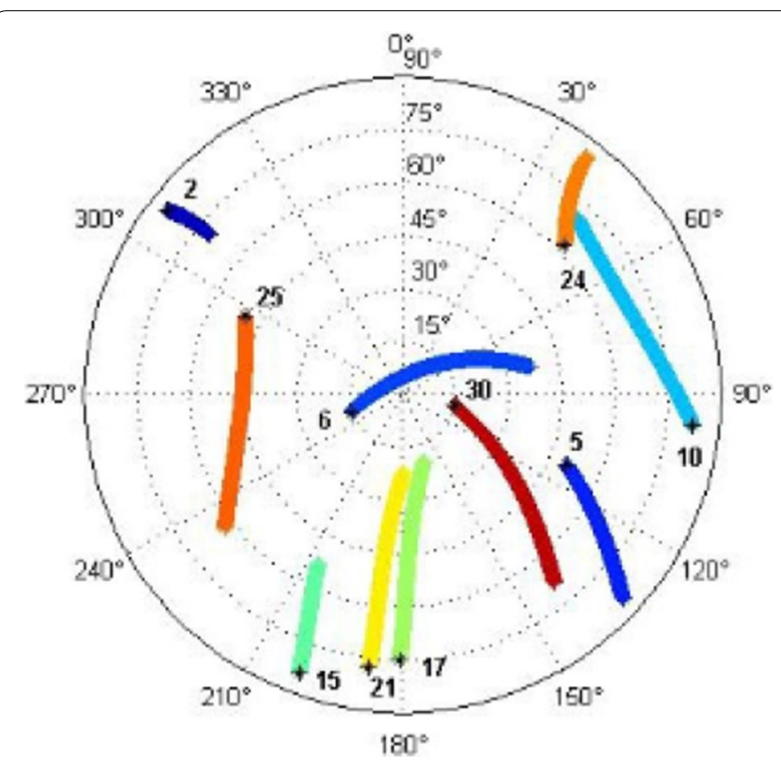

Fig. 7 Skyplot corresponding to the Seewinkel network. PRN6 is taken as the reference satellite for the computation of the DD

\section{Elimination of signal components}

After preprocessing the DD, the second step consists of extracting the noise from the multipath signal. Consequently, we firstly compute the EMD of the preprocessed DD residuals (see Fig. 8, right). The visual inspection confirms that the first four IMFs are more likely to contain the high-frequency noise of the residuals; the latter may be white, coloured or a combination of both. On the contrary, higher modes should correspond to the multipath envelope or unmodelled periodic component.

After having decomposed the noise into IMF, we perform the $h h t$ transformation and compute the $\log _{2}$ of the mean of the energy for each IMF. We estimate a slope of -0.95 for the line between the $\log _{2}$ variance between indices 2-4 by regression (Fig. 9, left, red line). The corresponding domains of exclusion corresponding to $3 \Delta$ are additionally depicted as dotted blue lines: They allow us to include the IMF 5 in the noise, whereas IMF 6 and 7 have to be eliminated prior to further analysis. The slope is recomputed by adding IMF 5 and reaches -0.90 , highlighting a high contamination with WN. However, the high negative slope does not speak in favour of a high contamination with $\mathrm{FN}$ at high frequencies.

\section{Filtering the WN}

In a second step, the multipath-free time series is further filtered by means of an adequate recombination of the EMD. A coefficient of $R_{\mathrm{WN}}=0.35$ was estimated. The time series obtained $S_{\mathrm{AN}}(t)$ is plotted in Fig. 9 (right, up) together with its psd estimated using the pwelch function of Matlab (Fig. 9, right, bottom). 

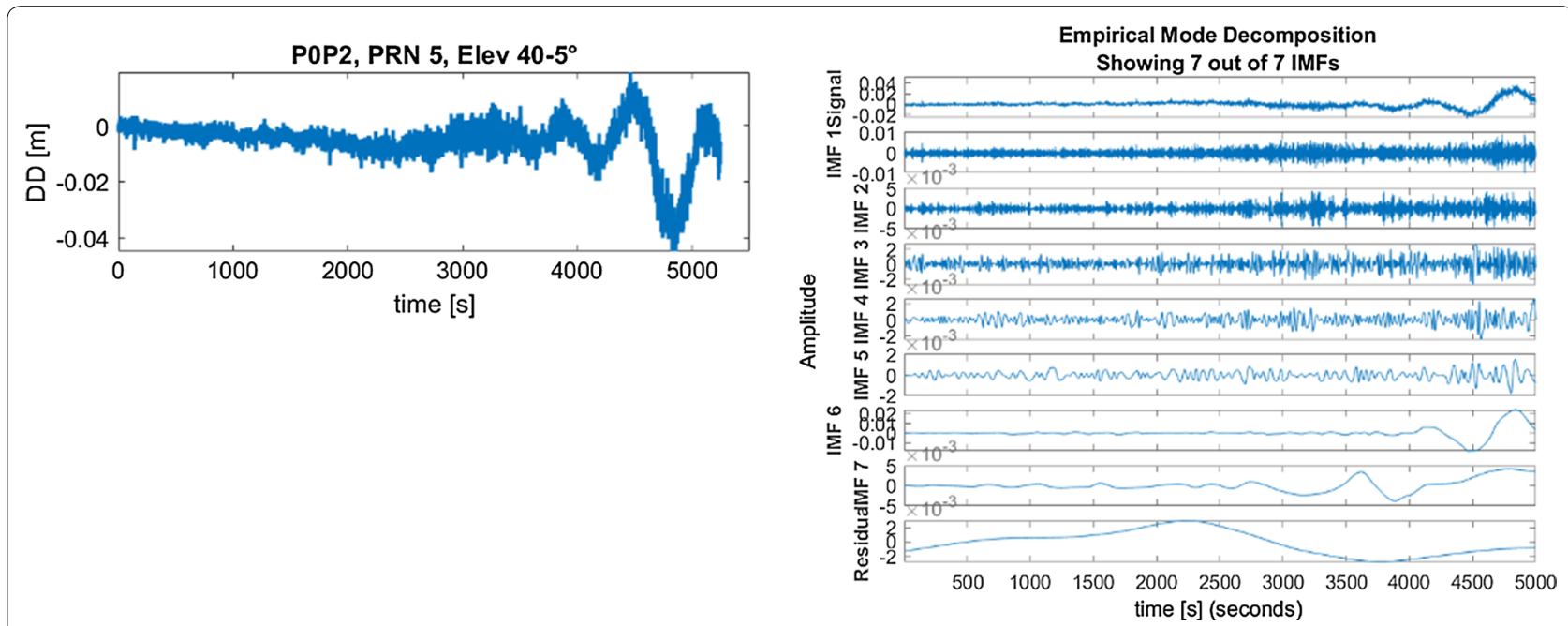

Fig. 8 Left: DD residuals in [m] for PRN5, baseline POP2. Right: the corresponding EMD decomposition using seven modes, pchip and a threshold of $1 e^{-6}$
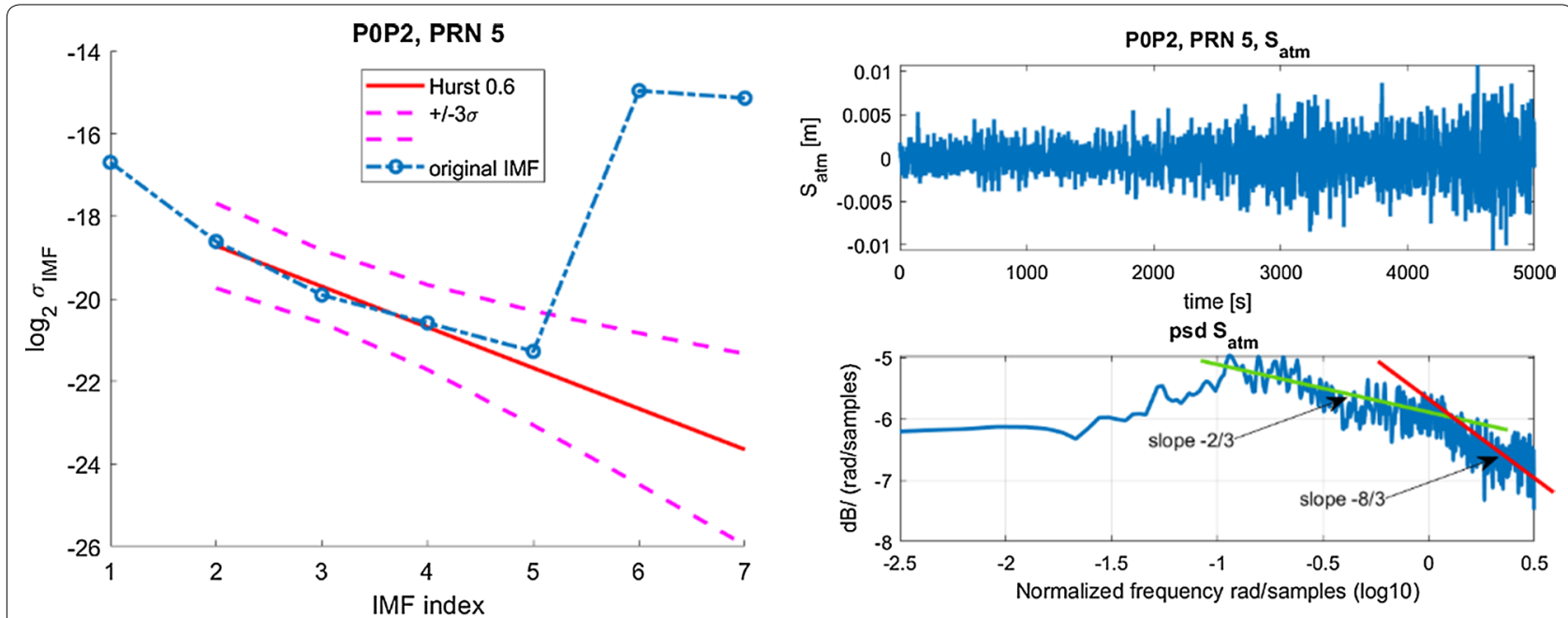

Fig. 9 Processing steps to identify atmospheric noise. Left: $\log _{2}$ variances of the IMF versus the IMF index. The red straight line corresponds to the $\mathrm{fGn}$ with $H=0.6$, i.e. the best fit for $\mathrm{IMF}_{2-4}$. The magenta line defines the domain of exclusion. The discrete blue dotted line represents the log 2 variance for the original observations. Right: top: the filtered time series as combination of the IMFs kept and at the bottom, its corresponding psd with the expected atmospheric slopes $-2 / 3$ and $-8 / 3$

We identify a psd corresponding to a Matérn process visually, by the typical damping at low frequency. Two slopes can be clearly identified, as well as their corresponding cut-off frequencies.

We use the debiased Whittle maximum likelihood to estimate these parameters. We found an outer scale length $L_{0}=3900 \mathrm{~m}$ and a separation distance $\rho_{\text {ray }}=5.1 \mathrm{~m}$, corresponding to the transition $-2 / 3$ and $-8 / 3$.

\section{Interpretation of the results}

We note that the outer scale length of turbulence corresponds to a plausible size of the eddies at the tropospheric height. At that height, we guessed, from physical consideration on electromagnetic wave propagation in random medium, that turbulence affects the GNSS signals. We also estimated the mean Euclidian distance between the GPS rays of PRN 5 and the reference satellite (PRN 6) at that height during the batch of 5050 epochs under consideration. The mean separation distance 
reaches a value of $3310 \mathrm{~m}$, which is coherent with the estimated cut-off length $L_{0}$, i.e. the maximum size of the eddies that affect GPS phase differences.

The cut-off separation distance at high frequency is slightly more difficult to interpret but could be linked, following Wheelon (2001 chapter 6), with the Euclidian distance between the rays from PRN 5 and 6 near the ground. This value is approximately $\rho_{\text {eff }}=4.9 \mathrm{~m}$ at a height of $2.5 \mathrm{~m}$ above the ground, a value close to the cutoff distance estimated. Clearly, this is only an approximation as in that case $v_{\text {eddies }}$ should not be approximated by the geostrophic wind velocity, leading to an even smaller value of $\rho_{\text {ray }}$.

Note on the estimation error an error of $\Delta \alpha=0.01$ for $\log _{10}(\alpha)=-1 \quad$ translates to $\left|\Delta L_{0}\right|=\left|\frac{2 \pi v_{\text {eddies }}}{\alpha^{2}} \Delta \alpha\right| \approx 50 \mathrm{~m}$, which is less than $5 \%$ of the nominal value, considering $L_{0}$ around $4000 \mathrm{~m}$. We consider this uncertainty as acceptable, regarding the unknown and relatively high value of the outer scale length. An error of $\Delta \alpha=0.01$ for $\log _{10}(\alpha)=0$ translates to $\left|\Delta L_{0}\right| \approx 0.5 \mathrm{~m}$, i.e. $\rho_{\text {ray }}$ is slightly more difficult to estimate with a high accuracy.

\section{Baselines POP2 and POP8 Results}

In this section, we propose to extend the analysis of the preprocessed residuals for all visible satellites using observations from the first and second batch of $2 \mathrm{~h}$ of observations for two stations P2 and P8. These two stations were chosen to compare the impact of increasing distance on the parameters estimated, i.e. to validate the assumption made in "Modelization of the power spectrum for phase differences" section about the nondependency of the results regarding the baseline length. Other results are not presented due to their similarities and for the sake of briefness, but can be made available on demand.

We follow the two-step methodology detailed in the previous section: (i) extracting the multipath component and (ii) eliminating the $\mathrm{WN}$ component. The results are presented in Table 1. We add the results of P0P2 for the ionospheric-free linear combination in Table 2.

\section{Comments}

Outer scale length of turbulence $L_{0} \quad$ Table 1 highlights that the outer scale length of turbulence $L_{0}$ can be estimated between 3500 and $3900 \mathrm{~m}$ from the GPS double difference phase power spectrum. This value corresponds approximately to the median value over all satellites and epochs of the separation distance, which reaches $4050 \mathrm{~m}$ for the first batch of observations and $4120 \mathrm{~m}$ for the second one, computed at the tropospheric height of $1000 \mathrm{~m}$. Thus, a plausible interpretation is that $L_{0}$ may correspond to the maximum size of eddies that act as correlating the phase difference. The low-frequency cut-off parameter is dependent neither on the elevation of the satellites nor on the baseline length, which is physically followable.

The ionospheric-free linear combination (Table 2) does not show any discrepancy with the previous results: the estimated mean value of $L_{0}$ is close to the

Table 1 Results for the Seewinkel network baselines POP2 and P0P8, the first batch of observations starting at epoch 20,700 , the second one starting at 27,800

\begin{tabular}{|c|c|c|c|c|c|c|c|}
\hline & PRN5 & PRN10 & PRN17 & PRN21 & PRN24 & PRN25 & PRN30 \\
\hline \multicolumn{8}{|l|}{1 st batch } \\
\hline \multicolumn{8}{|l|}{ POP2 } \\
\hline$L_{0}$ & 3900 & 3530 & 3730 & 3700 & 3690 & 3850 & 3960 \\
\hline$\rho$ & 5.1 & 5.3 & 5.3 & 4.9 & 5.2 & 5.1 & 5.3 \\
\hline \multicolumn{8}{|l|}{ POP8 } \\
\hline$L_{0}$ & 3470 & 3900 & 3700 & 3900 & 3570 & 3840 & 3540 \\
\hline$\rho$ & 5.0 & 5.4 & 6.8 & 5.3 & 5.1 & 5.1 & 6.9 \\
\hline $\begin{array}{l}\text { Mean separation distance } \\
h=1.5 \mathrm{~m}\end{array}$ & 4.9 & 4.6 & 2.3 & 2.7 & 6.2 & 3.1 & 1.5 \\
\hline \multicolumn{8}{|l|}{ 2nd batch } \\
\hline \multicolumn{8}{|l|}{ POP2 } \\
\hline$L_{0}$ & 3510 & 3920 & 3670 & 3830 & 3770 & 3575 & 3580 \\
\hline$\rho$ & 4.9 & 4.0 & 5.2 & 6.4 & 6.3 & 6.1 & 8.8 \\
\hline \multicolumn{8}{|l|}{ POP8 } \\
\hline$L_{0}$ & 3620 & 3780 & 3945 & 3595 & 3875 & 3780 & 3440 \\
\hline$\rho$ & 5.2 & 5.1 & 5.3 & 6.2 & 5.1 & 5.3 & 6.8 \\
\hline
\end{tabular}

$L_{0}$ and $\rho$ are expressed in $\mathrm{m}$. All values given for the outer scale length are approximated to the nearest $5 \mathrm{~m}$. We also give the value of the separation distance at a height of $1.5 \mathrm{~m}$ 
Table 2 Results for the Seewinkel network baseline POP1, one batch of observations starting at epoch 20,700

\begin{tabular}{llllllll}
\hline & PRN5 & PRN10 & PRN17 & PRN21 & PRN24 & PRN25 & PRN30 \\
\hline P0P1 & & & & & & \\
Lo & 2665 & 3930 & 3930 & 3500 & 4440 & 3950 & 3550 \\
$\rho$ & 2.9 & 6.1 & 5.7 & 5.9 & 3.8 & 5.6 & 5.9 \\
\hline
\end{tabular}

lonospheric-free linear combination

$L_{0}$ and $\rho$ are expressed in $\mathrm{m}$. All values given for the outer scale length are approximated to the nearest $5 \mathrm{~m}$

value found without eliminating the impact of the ionosphere. This confirms that the outer scale length in the ionosphere is probably much higher than in the troposphere, so that it cannot be detected in the relatively short time series. Moreover, its impact on the phase difference, mainly due to plasma irregularities (Rino 1979), is much smaller in magnitude than the one of the tropospheric turbulence.

The separation distance or cut-off at high frequencies The cut-off $\rho_{\text {ray }}$ reaches values between 4 and $8 \mathrm{~m}$ for all satellites, and its mean is close to $5 \mathrm{~m}$. It could be related to the separation distance between two rays computed near the ground, at a height of 1-2 m. It has some similarities with values found for optical turbulence (Coulman and Vernin 1991). However, we propose not to over-interpret this value, which is difficult to estimate with a high reliability since it does not correspond to a saturation of the power spectrum: the slope transition is not straightforward to identify. A bound within the expected range of frequencies for the estimation with Whittle maximum likelihood is necessary. Visually, $\rho_{\text {ray }}$ can be considered as nearly constant for all baseline lengths and satellites. It is not affected by the elimination of ionospheric effects (Table 2), as the latter should mainly be detected at low frequencies. Although the values of Table 2 are slightly higher than for Table 1 with ionospheric impact, they should be weighted by the fact that the ionosphere-free linear combination contains a strong WN component. The latter cannot be fully eliminated and impacts the determination of the cut-off at high frequencies.

The non-dependency with the baseline length is coherent with our assumption that a mean phase can be considered for medium baseline lengths (up to $30 \mathrm{~km}$ ) to compute the phase spectrum of the double differences. The low dependency with the elevation of the satellite is justified by the small batch lengths, making it difficult if not impossible to calculate an accurate temporal or, at least, batch-wise determination of this parameter. Further analysis and experiments are planned with highquality observations from single differences to validate a potential elevation dependency, as well as dependency with the wind vector.

\section{Conclusion}

A knowledge of the stochastic properties of GNSS phase difference observations is mandatory to get a reliable estimation of the position and for further processing implying test statistics. Any misspecification can lead to over- or underestimation of the precision. The study of the noise can be performed by analysing the residuals from DD processing of GNSS phase observations. In addition to WN or/and FN, it is expected that atmospheric noise will correlate with the observations for medium baseline lengths. These typical correlations come from the path of the GNSS signals through the atmosphere. They result from plasma irregularities in the ionosphere and turbulent structures called eddies in the troposphere. The latter source is considered to be stronger for GNSS phase observations and occurs in the loosely called free atmosphere. There, the reorganization of the eddies is less intense than near the ground, remaining, however, linked to strong values of the structure constant and a non-negligible energy. Using concepts from the turbulence theory, the psd of phase differences for microwaves in the turbulent troposphere can be modelled as a Matérn model modulated by a sinus term. Consequently, the spectrum of the phase difference residuals should present two slopes:

- $-8 / 3$ at high frequencies followed by

- a saturating $-2 / 3$ power law noise. The cut-off frequency of saturation corresponds to that of a damped Matérn process. This value can be related to the outer scale length of turbulence, i.e. the maximum size of the eddies acting as correlating the phase measurements, from physical consideration of the anisotropic turbulence in the free atmosphere.

These parameters can be estimated using the debiased Whittle maximum likelihood, which has the main advantage of being less biased for short samples than the normal maximum likelihood estimator. Estimating the parameters, as, e.g., the outer scale length leads to a more reliable results than finding slope intersections visually.

Prior to the estimation of the noise parameters, the residuals have to be filtered from WN and additional 
low frequency effects, such as multipath. In this contribution, we developed a strategy to extract the tropospheric noise from phase DD residuals by revisiting the EMD in an alternative way, which could be called "designalling the noise". We successfully applied our methodology to GPS observations from medium baselines from a network called "Seewinkel", specifically designed for the analysis of the impact of turbulence on GPS phase observations from DD phase residuals. We could estimate the threshold frequencies from a saturating two-slopes power spectrum. On the selected day and selected area, there were dynamic conditions on the periphery of upper-air anticyclone to the west. Given those conditions, the obtained results for outer scale turbulence between 3.5 and $4 \mathrm{~km}$ are plausible. We found furthermore that this value was related to the separation distance between GNSS rays, computed at the tropospheric height, where the main impact of the turbulence on GNSS observations is expected.

Our findings validate that:

- the extraction of the tropospheric noise from GPS phase residuals can be performed with a high reliability; the ionospheric-free linear combination confirmed the result,

- it is possible to derive physically plausible turbulence parameters-such as the outer scale length of turbulence-that can be linked with meteorological conditions,

- the stochastic model for GNSS phase measurements can be modelled, i.e. without empirical iterative computation, thus avoiding the overestimation of the precision.

We further computed the cut-off at high frequencies. The value obtained was found to be nearly constant for all satellites and baseline lengths and close to $5 \mathrm{~m}$, a value that is probably related to the separation distance computed near the ground.

Besides being useful in meteorology, GNSS phase observations can be used to analyse the turbulence in the atmosphere, with the restriction that the parameters may not be as accurate as with dedicated sensors.

Further analysis is planned to confirm our finding by analysing data sets from high accurate single difference observations. We expect to find a dependency of the cut-off frequency on the elevation of the satellites by a batch-wise estimation of the parameters.

\section{Abbreviations}

AN: Atmospheric noise; DD: Double differencing; EMD: Empirical mode decomposition; fBm: Fractional Brownian motion; fGn: Fractional Gaussian noise; FN: Flicker noise; GNSS: Global Navigation Satellite System; GPS: Global
Positioning System; H: Hurst exponent; hht: Huang-Hilbert Transform; IMF: Intrinsic mode functions; LS: Least squares; psd: Power spectrum density; WN: White noise.

\section{Acknowledgements}

The Seewinkel network was initially analysed during a Feodor-Lynen fellowship (A.v. Humboldt foundation) of the second author at TU Graz with Fritz K. Brunner as his host.

\section{Authors' contributions \\ GK developed the procedure to extract the atmospheric noise based on EMD decomposition and made the analysis of the Seewinkel network observations. She wrote the manuscript. SS discussed the results and contributes to the part on GPS processing as well as to the Seewinkel network description, on which he previously worked. Both authors read and approved the final manuscript.}

\section{Authors' information}

GK is currently working at the Geodetic Institute $(\mathrm{GlH})$ at the Leibniz University Hannover. Her main topics are stochastic modelization, with a focus on correlations. She works with GPS and terrestrial laser scanners observations, as well as on surface modelling and data approximation with B-spline surfaces.

SS is professor for positioning and navigation at the Institute für Erdmessung (IfE) at the Leibniz University Hannover.

\section{Funding}

Open Access funding enabled and organized by Projekt DEAL. This study is supported by the Deutsche Forschungsgemeinschaft under the project KE2453/2-1, as a pre-step to the analysis of atmospheric noise from a terrestrial laser scanner.

\section{Availability of data and materials}

The datasets used and/or analysed during the current study are available from the corresponding author on reasonable request.

\section{Ethics approval and consent to participate}

Not applicable.

\section{Consent for publication}

Not applicable.

\section{Competing interests}

The authors declare that they have no competing interests.

\section{Author details}

${ }^{1}$ Geodetic Institute, Leibniz Universität Hannover, Nienburger Str. 1, 30167 Hannover, Germany. ${ }^{2}$ Institut Für Erdmessung, Leibniz Universität Hannover, Schneiderberg 50, 30167 Hannover, Germany.

Received: 6 August 2020 Accepted: 4 November 2020

Published online: 04 December 2020

\section{References}

Bahreyni B (2008) Fabrication and design of resonant microdevices, vol 3. Micro \&amp; nano technologies. W. Andrew Inc, Norwich

Beutler G, Bauersima I, Gurtner W, Rothacher M, Schildknecht T, Mader GL, Abell MD (1987) Evaluation of the 1984 Alaska global positioning system campaign with the Bernese GPS Software. J Geophys Res 92(B2):1295-1303

Bevis M, Businger S, Chiswell S, Herring TA, Anthes RA, Rocken C, Ware RH (1994) GPS meteorology: mapping zenith wet delays onto precipitable water. J Appl Meteorol 33:379-386

Bos MS, Fernandes RMS, Williams SDP et al (2008) Fast error analysis of continuous GPS observations. J Geodesy 82:157-166

Buscher DF, Armstrong JT, Hummel CA et al (1995) Interferometric seeing measurements on Mt. Wilson: power spectra and outer scales. Appl Opt 34:1081-1096

Champollion C, Masson F, Van Baelen J et al (2004) GPS monitoring of the tropospheric water vapour distribution and variation during the 9 September 
2002 torrential precipitation episode in the Cévennes (southern France). J Geophys Res. https://doi.org/10.1029/2004JD004897.

Champollion C, Drobinski P, Haeffelin M et al (2009) Water vapour variability induced by urban/rural surface heterogeneities during convective conditions. QJR Meteorol Soc 135:1266-1276

Choy S, Wang C, Zhang Ket al (2013) GPS sensing of precipitable water vapour during the March 2010 Melbourne storm. Adv Space Res 52:1688-1699

Coulman CE, Vernin J (1991) Significance of anisotropy and the outer scale of turbulence for optical and radio seeing. Appl Opt 30:118-126

Dach R, Brockmann E, Schaer S et al (2009) GNSS processing at CODE: status report. J Geodesy 83:353-365

Dach R, Schaer S, Arnold D, Kalarus MS, Prange L et al (2020) CODE final product series for the IGS. Astronomical Institute, University of Bern, Bern

Dodson AH, Shardlow PJ, Hubbard LCM et al (1996) Wet tropospheric effects on precise relative GPS height determination. J Geodesy 70:188-202

Douša J, Vaclavovic P (2014) Real-time zenith tropospheric delays in support of numerical weather prediction applications. Adv Space Res 53:1347-1358

Flandrin P, Rilling G, Gonçalvès P (2004) Empirical mode decomposition as a filter bank. IEEE Signal Process Lett 11:112-114

Flandrin P, Gonçalvès P, Rilling G (2005) EMD equivalent filter banks, from interpretation to applications. In: Huang NE, Shen SSP (eds) Hilbert-Huang transform and its applications, vol 5. World Scientific, Singapore, pp 57-74

Guerova G, Jones J, Douša J et al (2016) Review of the state of the art and future prospects of the ground-based GNSS meteorology in Europe. Atmos Meas Tech 9:5385-5406

Halsig S, Artz T, Iddink A et al (2016) Using an atmospheric turbulence model for the stochastic model of geodetic VLBI data analysis. Earth Planets Space 68:106. https://doi.org/10.1186/s40623-016-0482-5

Hinder R (1972) Fluctuations of water vapour content in the troposphere as derived from interferometric observations of celestial radio sources. J Atmos Terr Phys 34:1171-1186

Hofmann-Wellenhof B, Lichtenegger H, Wasle E (2008) GNSS—global navigation satellite systems. GPS, GLONASS, Galileo, and more. Springer, New York

Huang NE, Shen SSP (2005) Hilbert-Huang transform and its applications, vol 5. Interdisciplinary mathematical sciences. World Scientific, New Jersey

Huang NE, Shen Z, Long SR et al (1998) The empirical mode decomposition and the Hilbert spectrum for nonlinear and non-stationary time series analysis. Proc R Soc Lond A 454:903-995

Hirrle A (2017) Zur Reduzierung des mehrwegebedingten GNSS Trägerphasenmessfehlers durch Anwendung der Hilbert-Huang-transformation auf Signalqualitätsparameter. Saechsische Landesbibliothek-Staats- und Universitaetsbibliothek Dresden

Ishimaru A (2017) Electromagnetic wave propagation, radiation, and scattering, 2nd edn. Wiley, Hoboken

Johnston G, Riddell A, Hausler G (2017) The international gnss service. In: Teunissen PJ, Montenbruck O (eds) Springer handbook of global navigationsatellite systems, Springer handbooks. Springer, Cham, pp 967-982. https://doi.org/10.1007/978-3-319-42928-1_33

Kargoll B, Omidalizarandi M, Loth I et al (2018) An iteratively reweighted leastsquares approach to adaptive robust adjustment of parameters in linear regression models with autoregressive and t-distributed deviations. J Geodesy 92:271-297

Kermarrec G, Schön S (2014) On the Mátern covariance family: a proposal for modeling temporal correlations based on turbulence theory. J Geodesy 88:1061-1079

Kermarrec G, Ren Le, Schön S (2018) On filtering ionospheric effects in GPS observations using the Matérn covariance family and its impact on orbit determination of Swarm satellites. GPS Solut 22:66

Klos A, Hunegnaw A, Teferle FN et al (2018) Statistical significance of trends in Zenith Wet Delay from re-processed GPS solutions. GPS Solut 22:51

Kolmogorov AN (1941) Dissipation of energy in locally isotropic turbulence. Proc USSR Acad Sci 32:16-18 (in Russian)

Kopsinis Y, McLaughlin S (2009) Development of EMD-based denoising methods inspired by wavelet thresholding. IEEE Trans Signal Process 57(4):1351-1362

Leick A, Rapoport L, Tatarnikov D (2015) GPS satellite surveying, 4th edn. Wiley, Hoboken

Li G, Deng J (2013) Atmospheric water monitoring by using ground-based GPS during heavy rains produced by TPV and SWV. Adv Meteorol 2013:1-12

Lilly JM (2020) jLab: a data analysis package for Matlab, v. 1.6.7. https://www.jmlil ly.net/jmlsoft.html. Accessed 21 July 2020
Lilly JM, Sykulski AM, Early JJ et al (2017) Fractional Brownian motion, the Matérn process, and stochastic modeling of turbulent dispersion. Nonlinear Processes Geophys 24:481-514

Mandelbrot BB, van Ness JW (1968) Fractional Brownian motions, fractional noises and applications. SIAM Rev 10:422-437

Montillet J-P, Tregoning P, McClusky S et al (2013) Extracting white noise statistics in GPS coordinate time series. IEEE Geosci Remote Sens Lett 10:563-567

Myrup LO (1969) Turbulence spectra in stable and convective layers in the free atmosphere. Tellus 21:341-354

Naudet CJ (1996) Estimation of troposperic fluctuations using GPS data, Pasadena, $C A$

Odijk D (2002) lonosphere-free phase combinations for modernized GPS. J Surv Eng 129:165-173

Percival DB, Walden AT (1993) Spectral analysis for physical applications. Multitaper and conventional univariate techniques, reprinted with corrections. Cambridge University Press, Cambridge

Rino CL (1979) A power law phase screen model for ionospheric scintillation: 1. Weak Scatter. Radio Sci 14:1135-1145

Saastamoinen J (1973) Contributions to the theory of atmospheric refraction. Bull Geodesique 107:13-34

Satirapod C, Rizos C (2005) Multipath mitigation by wavelet analysis for GPS base station applications. Surv Rev 38:2-10

Schön S, Brunner FK (2008) Atmospheric turbulence theory applied to GPS carrier-phase data. J Geodesy 82:47-57

Schön S, Pham H, Kersten T, Leute J, Bauch A (2016) Potential of GPS Common Clock Single-differences for Deformation Monitoring. J Appl Geod 10(1):45-52. https://doi.org/10.1515/jag-2015-0029

Stoev S (2020) Simulation of fractional Gaussian noise *EXACT* . https://www. mathworks.com/matlabcentral/fileexchange/19797-simulation-of-fract ional-gaussian-noise-exact. MATLAB Central File Exchange. Accessed 21 July 2020

Stotskii AA, Stotskaya IM (1992) Analysis of tropospheric pathlength fluctuations using geostationary satellite observations. Astron Astrophys Trans 2:327-339

Stull RB (1994) An introduction to boundary layer meteorology, vol 13. Atmospheric Sciences Library. Kluwer, London

Sykulski AM, Olhede SC, Guillaumin AP et al (2019) The debiased Whittle likelihood. Biometrika 106:251-266

Taebi A (2017) Noise cancellation from vibrocardiographic signals based on the ensemble empirical mode decomposition. J Appl Biotechnol Bioeng 2(2):49-54

Tatarskii VI (1971) The Effects of the turbulent atmosphere on wave propagation. (Rasprostranenie voln vturbulentnol atmosfere). Israel Program for Scientific Translations; Reproduced by National Technical Information Service U.S. Dept. of Commerce, Jerusalem, Springfield, VA

Taylor Gl (1938) The spectrum of turbulence. Proc R Soc A Math Phys 164:476-490

Teunissen PJG, Montenbruck O (eds) (2017) Springer handbook of global navigation satellite systems. Springer Handbooks. Springer International Publishing, Cham

Vennebusch M, Schön S (2011) Generation of slant tropospheric delay time series based on turbulence theory. In: Kenyon SC, Pacino MC, Marti U (eds) Geodesy for planet Earth. Proceedings of the 2009 IAG symposium, Buenos Aires, Argentina, 31 August-4 September 2009, vol 136. Springer, Berlin, pp 801-807

Wang T, Zhang M, Yu Q, Zhang H (2012) Comparing the applications of EMD and EEMD on time-frequency analysis of seismic signal. J Appl Geophys 83:29-34

Wheelon AD (2001) Electromagnetic scintillation. Cambridge University Press, Cambridge

Wu J (2009) Mitigation of GPS carrier phase multipath effects using empirical mode decomposition. In: Staff I (ed) 2009 international conference on information engineering and computer science. IEEE, New York, pp 1-4

Wu Z, Huang NE (2004) A study of the characteristics of white noise using the empirical mode decomposition method. Proc R Soc Lond A 460:1597-1611

Zhao Q, Yao Y, Yao W et al (2018) Real-time precise point positioning-based zenith tropospheric delay for precipitation forecasting. Sci Rep 8:7939

\section{Publisher's Note}

Springer Nature remains neutral with regard to jurisdictional claims in published maps and institutional affiliations. 